\title{
Semi-autonomous Telerobotic Manipulation: A Viable Approach for Space Structure Deployment and Maintenance
}

\author{
Young S. Park ${ }^{1}$, Hyosig Kang ${ }^{1}$, Thomas F. Ewing ${ }^{1}$, Eric L. Faulring ${ }^{2}$, Brian P. \\ DeJong ${ }^{2}$, Michael A. Peshkin ${ }^{2}$ J. Edward Colgate ${ }^{2}$ \\ ${ }^{1}$ Nuclear Engineering Division, Argonne National Laboratory, Argonne, IL 60439 \\ ${ }^{2}$ Department of Mechanical Engineering, Northwestern University, Evanston, IL 60208-3111 \\ (630) 252-5094,ypark@anl.gov
}

\begin{abstract}
Future space explorations necessitate manipulation of space structures in support of extra vehicular activities or extraterrestrial resource exploitation. In these tasks robots are expected to assist or replace human crew to alleviate human risk and enhance task performance. However due to the vastly unstructured and unpredictable environmental conditions, automation of robotic task is virtually impossible and thus teleoperation is expected to be employed. However teleoperation is extremely slow and inefficient. To improve task efficiency of teleoperation, this work introduces semi-autonomous telerobotic operation technology. Key technological innovations include implementation of reactive agent based robotic architecture and enhanced operator interface that renders virtual fixture.
\end{abstract}

\section{INTRODUCTION}

Future space exploration programs necessitate deployment and maintenance of large structures, such as space observatories and spaceships. Since space is a remote and dangerous place, robotic systems are expected to replace human astronauts in performing such tasks. However, due to the unstructured and unpredictable nature of the task environment, it is practically impossible to automate the robot operations in such a way that can reliably accommodate the unpredictable task conditions. In this regards, a viable option would be to adopt teleoperation, in which robot operation relies solely on human perception-action. However, mainly due to the poor sensory display, teleoperation is extremely slow and inefficient process. Also, precision motion, as often required for structural assembly and disassembly, are difficult to accomplish through manual teleoperation. Such difficulties are evidenced by DOE's experiences of deploying robotic systems for decontamination and decommissioning (D\&D) of nuclear facilities (DOE/EM, 1998; Noakes, 2002). What is required is powerful operator aid which can expand the operator's ability to guide efficient and precise manipulation. In this regards, joint R\&D activities have been conducted at Argonne National Laboratroy and Northwestern University to facilitate two types of enhancements: semi-automatic teleoperation technologies that blends teleoperation with autonomous behaviors (Park 2004), and enhances user interface to attain efficient robotic actions (Faulring, 2004; Dejong, 2004). Such enhanced telerobotic manipulation technologies, although originally developed and demonstrated for D\&D (decontamination and decommissioning) of nuclear facilities, share common basis with the application needs in space programs. The purpose of this paper is to outline the recent development of such enhanced telerobotic operation technology at Argonne National Laboratory, and suggest its applicartion for tasks in space exploration.

\section{TELEAUTONOMY}

Tele-autonomy is achieved by blending human oversight of teleoperation with sensor-based autonomous operation. Autonomy can be implemented based on either deliberative or reactive architecture. Although well suited for structured and highly predictable environments, the deliberative architecture lacks the flexibility to cope with uncertain and unstructured environments. On the other hand, a reactive system is composed of a collection of behaviors that tightly link sensory inputs to motor actions. The flow of control and communication between each behavior is less restrictive, and autonomous control emerges from the interaction of multiple behaviors. A reactive 
system is more suitable for autonomy in unstructured and uncertain environment. Furthermore, due to its distributed nature, it exhibits incremental competency - more complex behaviors can be built and tested incrementally from elementary behaviors.

In our implementation, a reactive system is composed of motor agents that directly correlate sensory inputs to the manipulator's motor actions as introduced by (Arkin, 1991). As shown in Figure 1, the robot motion is determined as emergent response of multiple motor behaviors. Embedded within each motor agent is a perceptual agent that provides the perceptual information customized for the respective motor behavior.

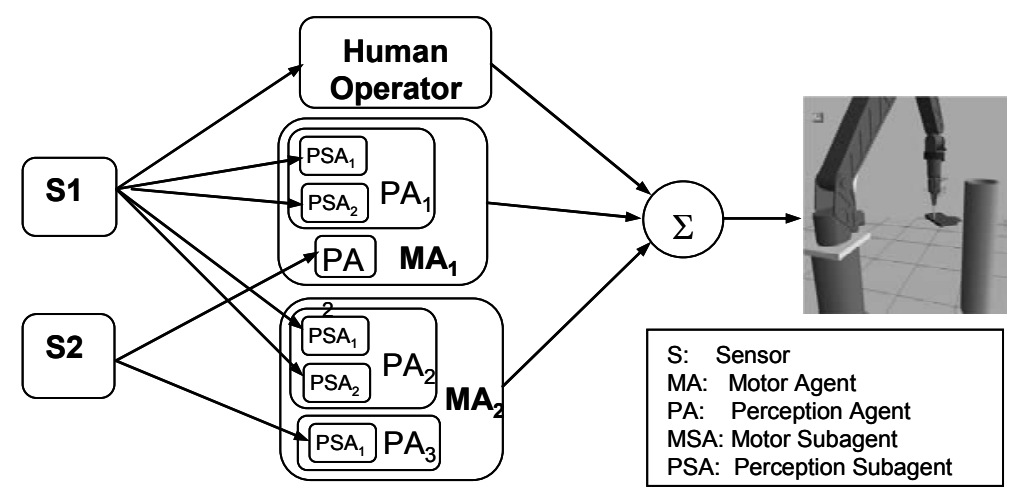

FIGURE 1. Reactive Robotic Architecture.

\section{Motor Agents}

From our experience of deploying robotic equipment for decontamination and dismantling (D\&D) of a research nuclear reactor, common manipulation tasks are composed of cutting pipes and bolts, tearing down graphite blocks, cutting reactor walls, unscrewing bolts, carrying waste baskets and loads, inspection, and others. By analyzing the nature of these tasks, it can be noted that various strategic tasks emerge from a few simpler common behavioral schemas, call it motor agents. Each motor agent is characterized by tightly linking sensory input to motor actions.

In our implementation, motor agents are encoded as mathematical functions, depicting as output the manipulator's end-effector motion, $\dot{\mathbf{x}}$, so that

$$
\dot{\mathbf{x}}=\left[\begin{array}{l}
\mathbf{v} \\
\mathbf{\Omega}
\end{array}\right],
$$

which is composed of linear velocity, $\mathbf{v}=\left[v_{\mathrm{x}}, v_{\mathrm{y}}, v_{\mathrm{z}}\right]$, and angular velocity, $\Omega=\left[\omega_{\mathrm{x}}, \omega_{\mathrm{y}}, \omega_{\mathrm{z}}\right]$.

Teleoperation, allows the human operator to provide internal bias to the control system.

Approach-goal, permits the robot to move toward a specified goal location, decelerating at close proximity to the goal location.

Align-tool, places and orients the tool in the direction normal to the surface of a part to be dismantled.

Dock, slowly moves the tool in the direction normal to the part surface until it is pressed against the part.

Move-along-path, moves the tool along a path generated from a perceptual agent, define-tool-path.

Maintain-attitude, provide bias to the manipulator trajectory in such a way that maintains offset distance away from or into the surface.

Escape, moves the tool in backward biased random directions to escape from a jammed condition.

Avoid-joint-limit, provide beneficiary motion to prevent approaching the joint limits of the manipulators. 


\section{Behavioral Assemblage and Coordination}

High-level behavioral assemblages can be composed from the primitive motor agents. In this work, behavioral assemblages are composed for the following abstract tasks.

Transport_tool: Human operator carries the tool, held by the robotic arms, from one location to a goal location marked by a structured beam projection described in the next section. Reactive agents assist human effort by providing bias motion to direct and align the tool to the goal location. Surprisingly, this task is most time consuming when performed by manual teleoperation, due to poor visual display.

Apply_tool: Tooling generally requires precise motion in a rather structured environment, and thus can benefit from automating the process. Based on partial knowledge of the task geometry and sensory information, the task trajectory is generated and updated, and reactive agents provide motions to track the trajectory, with task-specific motion constraints.

As shown in Figure 2, the responses of motor agents are individually adjusted by respective gains and aggregated, through behavioral coordination. How we assign the gains to individual behavioral response may define another high-level behavior of the robot system. For example, the following high-level behaviors further characterize the robot as following,

$$
\text { Robot (robust_robot, efficient_robot, cautious_robot) }
$$

Robust_robot puts more weight to teleoperation so that it is robust to adapt to unstructured environment. Efficient_robot places more weight to autonomous behaviors so that it is suitable for tasks in structured environment with higher degree of automation. For applications where safety is critical, cautious_robot limits the speed and acceleration and makes conservative choices in executing motions when safety is in doubt.

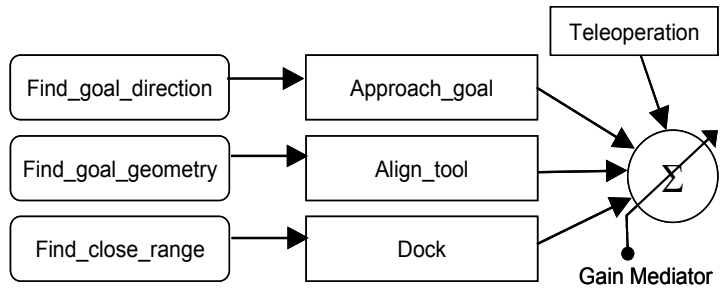

(a) Transport_Tool.

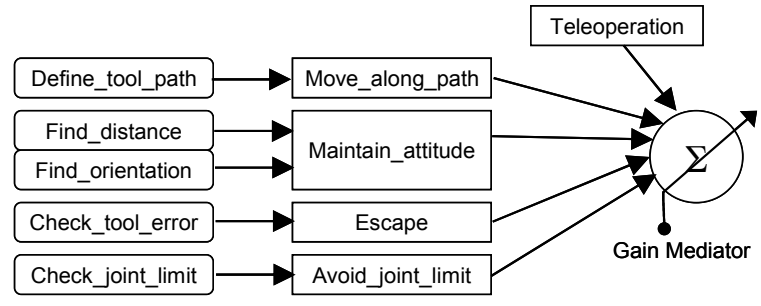

(b) Apply_Tool.

FIGURE 2. Behavioral Assemblages.

\section{Perceptual Agents}

The sensor system is composed of a structured-light system and proximity sensor array, as shown in Figure 3. A structured light system consisting of a laser beam projector and a camera provide pose information at mid-range (Park 2000). The beam pattern projected over an environmental object provides visual reference to both motor agents and the human operator. Also, the laser beam projection may guide where to focus attention in the visual search process, resulting in significant reduction in the computational complexity. By analyzing the distortions in the projected grid patterns, the location, orientation, and surface details of a part can be determined. An infrared proximity sensor array provides near-range measurement. In the development of a behavior-based robotic system, perception is viewed as a partner process with action. The need for motor control provides context for perceptual processing, whereas perceptual processing is simplified through the constraints of motor action. Taking this interplay into account, the following perceptual agents are devised.

grid_on: Flags if grid beam projection is on.

in mid_range: From the size of the grid image, make a coarse measurement of distance to the grid projection. Flags when the distance is within a preset value. 


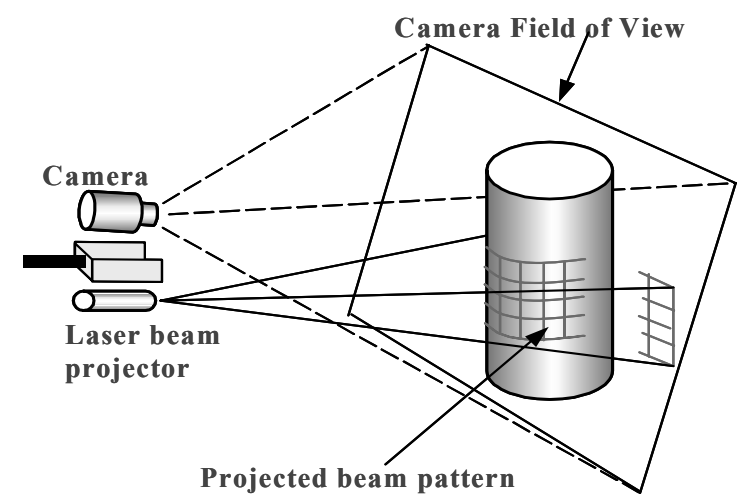

(a) Structured Light System.

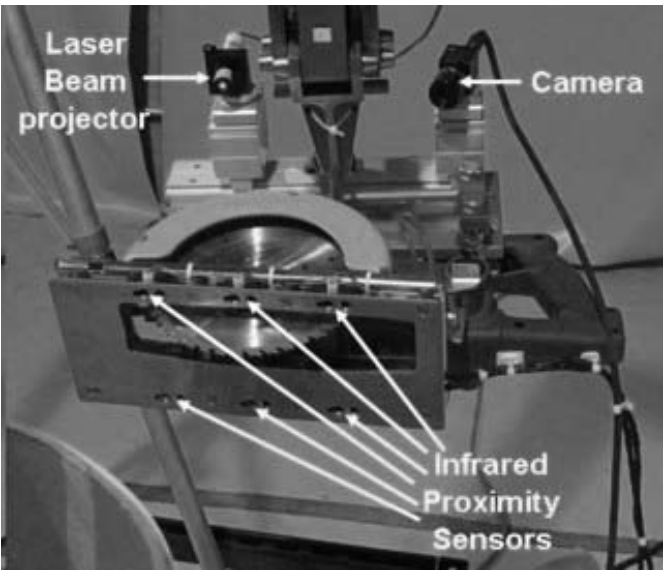

(b) Proximity Sensor Array.

FIGURE 3. Sensor Systems.

find_goal_direction: From the center position of the projected landmark pattern in the camera field of view, it determines the direction to the grid location.

find_precise_range: Make precise range measurement of the grid points using the structured light sensor as described in the previous section.

find_shape: Identify shape of an object from a range map.

find_goal_geometry: Estimate geometric parameters of an object of known geometry from a range map.

generate_tool_path: It generates desired tool trajectory from object geometric model and grid projection. The

result is stored in long-term memory.

\section{Experimental Operation: Teleautonomy}

To demonstrate usefulness of teleautonomy, experimental operations were performed, in which manipulator holds a circular saw with its gripper and cuts around the circumference of a pipe, a structure commonly encountered during D\&D operations. Circular saw is tool commonly used for sectioning nuclear reactor walls and pipes. Its operation requires maintaining precise alignment and reinsertion of tool blade, as well as commencing steady forward motion in the cutting direction. During a previous D\&D demonstration at a research reactor, CP-5, manipulation of this device was proven inefficient with manual teleoperation (DOE/EM, 1998). The semi-autonomous operation is
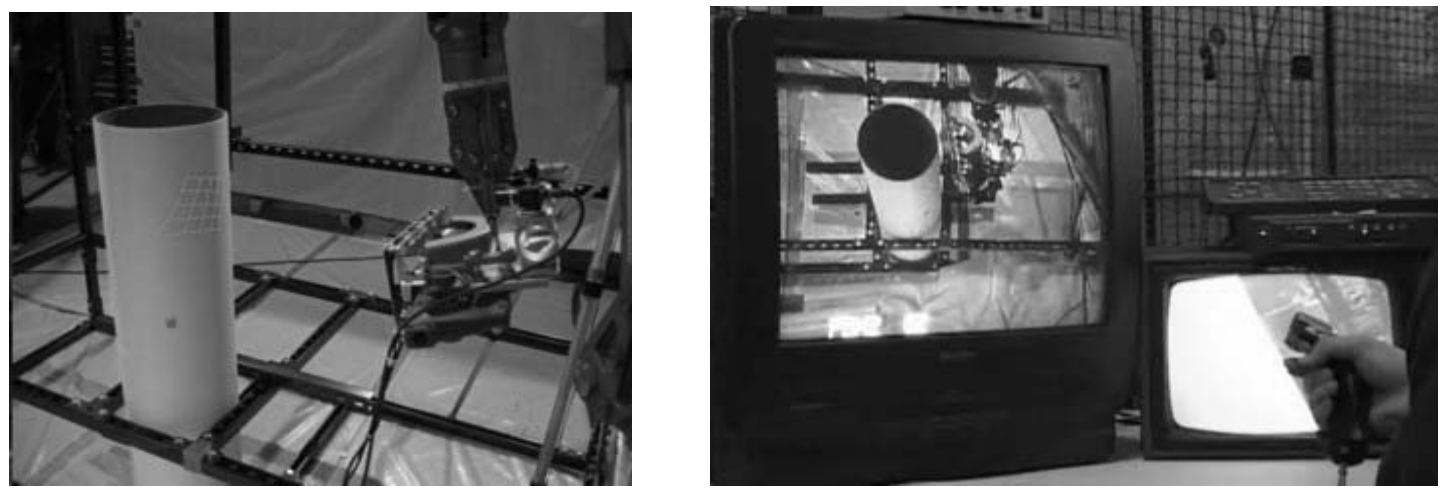

FIGURE 4. Experimental Operation: Teleautonomy. 
demonstrated using a telerobotic system consisting of a Schilling Titan 7F six degree-of-freedom manipulator, telerobotic control system, sensory system, and graphically augmented video display system. The telerobotic control system consists of a slave robot servo controller, master input device, and a control computer. The sensory system consists of multiple proximity sensors and structured light sensors, for which a dedicated computer is assigned. Two other computers are assigned for real-time video and graphic display. The multiple remote computer systems are interfaced through network, both TCP and UDP protocols are used.

Figure 5 shows the tool paths during both manual teleoperation and semi-automatic teleoperation. As can be seen in Fig. 5(A), manual operation resulted in poor trajectory control. It was difficult to align the tool to the work piece and maintain tool motion along a desirable tool path with proper orientation, and moreover, the process was extremely time-consuming. On the other hand, in teleautonomy, much more precise trajectory control was accomplished with help of autonomous behaviors, as can be seen in Figure 5(B). It was also observed that the semi-autonomous teleoperation was more effective in maintaining proper tool orientation during the approach and cutting process. Overall, teleautonomous operation enabled precise cutting to be performed in much shorter time, even by nontrained operators.

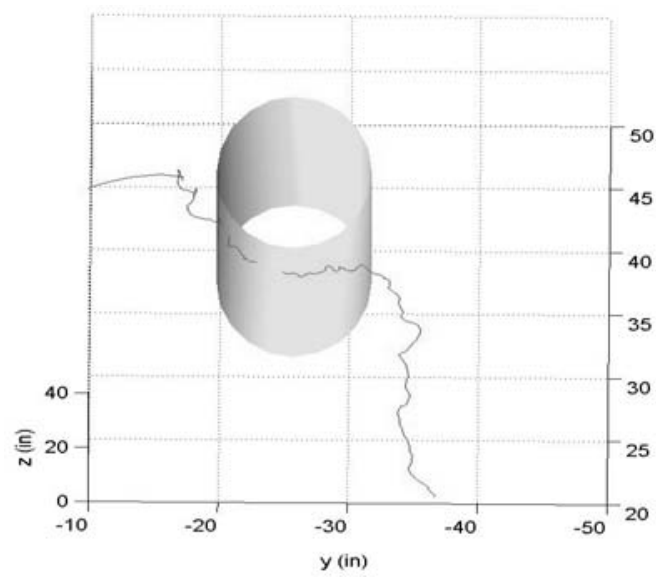

(a) Manual Teleoperation.

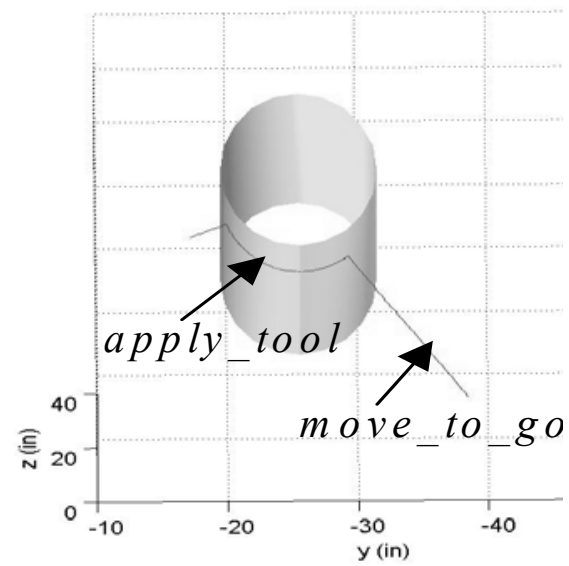

(b) Teleautonomous Operation.

FIGURE 5. Tool Trajectories During Experimental Teleoperation.

\section{ENHANCED OPERATOR INTERFACE}

Another enhancement of telerobotic operation addressed provision of effective operator interface. Central to this effort is the implementation of 'virtual fixture'. Virtual fixture is artificially generated surface overlaid on human percepts, thus providing passive constraint in such a way that guides human action in aid of task performance (Rosenberg 1994). The type of virtual fixture adopted in this is a virtual funnel, which guide the cutting tool toward the target pipe during the approach phase. The virtual funnel is defined with radius, $\varepsilon_{\mathrm{s}}$, at the start position, $\mathrm{p}_{\mathrm{s}}$ and $\varepsilon_{\mathrm{f}}$, at the end position, $p_{f}$, and the tunnel axis $n_{t}=p_{f}-p_{s}$. A cubic function can be used to determine the funnel radius, $\varepsilon_{\mathrm{t}}$, representing how to tolerate the translational deviation from the desired approach trajectory. The funnel radius $\varepsilon_{\mathrm{t}}$ at a given $t \in[0,1]$ is defined as

$$
\varepsilon_{\mathrm{t}}(\mathrm{t})=\varepsilon_{\mathrm{f}}+\left(\varepsilon_{\mathrm{s}}-\varepsilon_{\mathrm{f}}\right)\left(2 \mathrm{t}^{3}-3 \mathrm{t}^{2}+1\right) .
$$

As shown in Figure 6, the virtual funnel shrinks as it approaches the target position. The corresponding funnel radius represents how to tolerate the translational deviation from the desired approaching trajectories. As long as the tool remains inside the funnel, it will proceed toward the target position.

The virtual fixture may be presented as visually augmented display. The graphic display of virtual funnel as shown Figure is devised to assist in translational guidance. In order to assist the operator to align the tool orientation, a virtual aligning cone is also devised. The virtual cone can be represented as having its vertex at the origin of the tool 


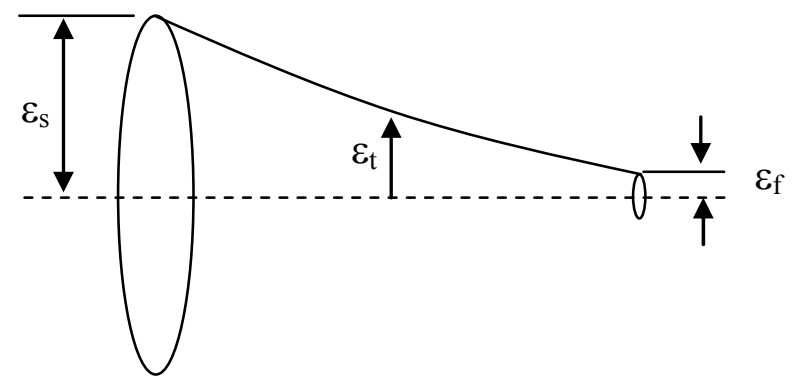

FIGURE 6. Virtual Funnel Geometry.

frame, the axis vector $\mathbf{a}$ aligned the desired direction vector $\mathbf{r}_{\mathbf{d}}$, and angle $\alpha$. Since $\mathbf{a}$ is aligned with $\mathbf{r}_{\mathbf{d}}$, the virtual cone angle $\alpha$ represents how to tolerate the rotational deviation from the desired direction vector. Hence, the operator can get the intuitive perception of the desired rotational motion from this geometrical guidance. Virtual cone can be concisely represented with the introduction of quaternion. With the given start orientation $\mathbf{q}_{\mathbf{s}}$ and the target orientation $\mathbf{q}_{\mathbf{f}}$, the spherical linear interpolation of quaternion at $t \in[0,1]$ yields

$$
\mathbf{q}_{\mathbf{t}}\left(\mathrm{t}, \mathrm{q}_{\mathrm{s}}, \mathrm{q}_{\mathrm{f}}\right)=\left(\mathrm{q}_{0}, \mathrm{q}\right)=\left\{\sin ((1-\mathrm{t}) \theta) \mathbf{q}_{\mathrm{s}}+\sin (\mathrm{t} \theta) \mathbf{q}_{\mathbf{f}}\right\} / \sin (\theta) \text {, where } \theta=2 \cos ^{-1}\left(\mathbf{q}_{\mathbf{s}} \cdot \mathbf{q}_{\mathbf{f}}\right) .
$$

Thus, the centered normal axis vectors of three virtual cones can be defined with orthonormal directional vectors from the following equivalent rotation matrix,

$$
\mathbf{R}=\left[\mathbf{r}_{1 \mathrm{~d}} \mathbf{r}_{2 \mathrm{~d}} \mathbf{r}_{3 \mathbf{d}}\right]=\mathbf{I}+(\sin \phi) \mathbf{S}+(1-\cos \phi) \mathbf{S}^{2},
$$

where $\phi=2 \cos ^{-1}\left(\mathrm{q}_{0}\right)$ and $\mathbf{S}=(\mathrm{q} /\|\mathrm{q}\|)^{\wedge}$.

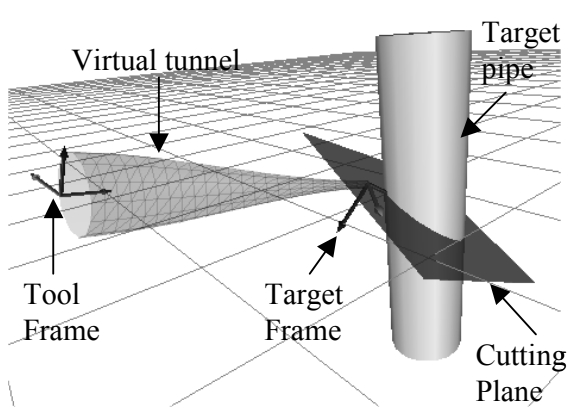

(a) Overview of Virtual Fixture.

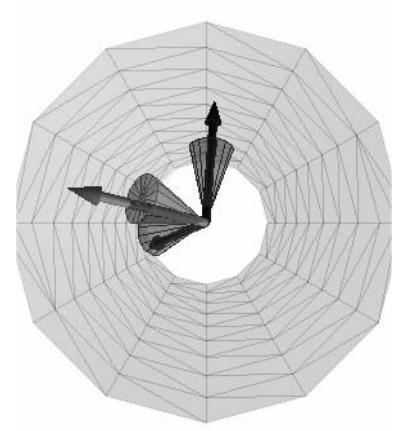

(b) Looking Into the Funnel.

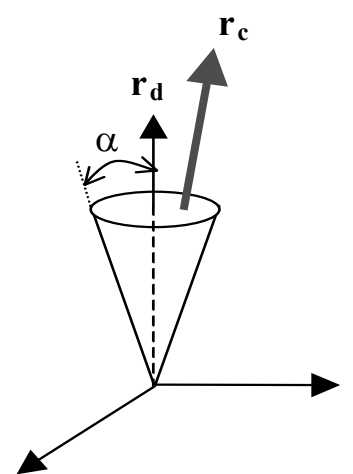

(c) Virtual Alignment Cone.

FIGURE 7. Visually Rendered Virtual Funnel.

To display the virtual fixture in haptic domain, a new hand controller is developed, based on Cobot technology (Faulring, 2004). Cobot is a proprietary technology capable of providing smooth yet extremely strong constraints through the use of non-holonomic constraints (Wannasuphoprasit, 1998, Peshkin, 2001). A steered wheel, un-powered about its rolling axis, creates a relationship between the two components of its linear velocity. Higher dimension cobots utilize varying geometries of rolling contacts. Cobots can either be operated in "free-mode," where the intent of the operator in the full dimension of the task space is followed completely, or in "virtual-surface" mode, where a lower dimensional surface that the task space guides the operator's intent tangent to that surface, and the nonholonomic constraints of the rolling wheels, not the torque of any actuators, prevent motion normal to the virtual surface. The design of this 6-DOF Cobotic Hand Controller utilizes the kinematics of a parallel platform introduced 
by (Merlet, 1992), which is illustrted in Figure 8. The proximal links are coupled by three degree-of-freedom universal joints to the distal links, and these in turn are coupled via two degree-of-freedom universal joints to an end-effector platform. A force sensor on the end-effector is used to determine the user's intent. Our addition to Merlet's kinematics has been to couple the six linear actuators to a central "power cylinder" through non-holonomic constraints. Linear actuation of the proximal links is achieved via a rotational to linear continuously variable transmission(CVT), namely a steered wheel. The angle of each wheel relates the linear velocity $v_{i}$ of each proximal link to the rotational velocity of the power cylinder $\omega$. When the wheels are steered such that their rolling axis is parallel to the power cylinder $\left(\phi_{\mathrm{i}}=0\right)$, a ratio $v_{\mathrm{I}} / \omega=-\mathrm{r} \tan \left(\phi_{\mathrm{i}}\right)=0$ is set. If the wheels are steered either direction from $\phi_{\mathrm{i}}=0$, ratios between \pm infinity can be achieved. In practice, wheel slip limits this range. It is also evident, that turning all six wheels to $\phi_{\mathrm{i}}=0$ locks the six actuators, and turning them to $\phi_{\mathrm{i}}=\pi / 2$ completely decouples the actuators from the cylinder's velocity, although the cylinder would then be unable to turn.

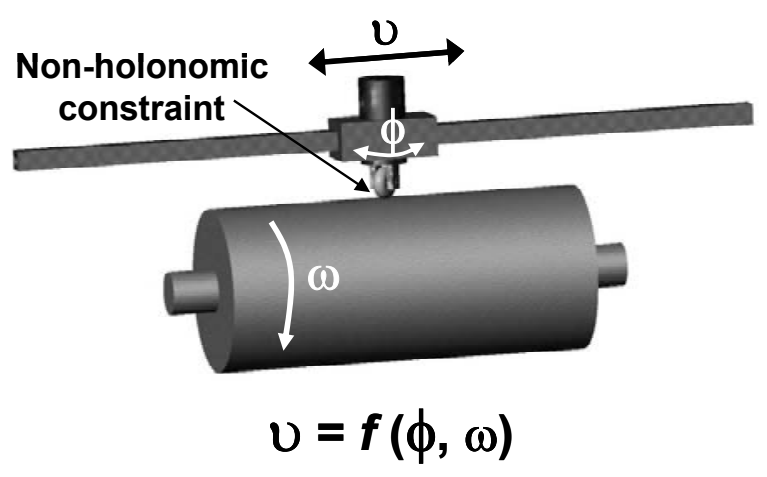

(a) Continuously Variable Transmission (CVT).

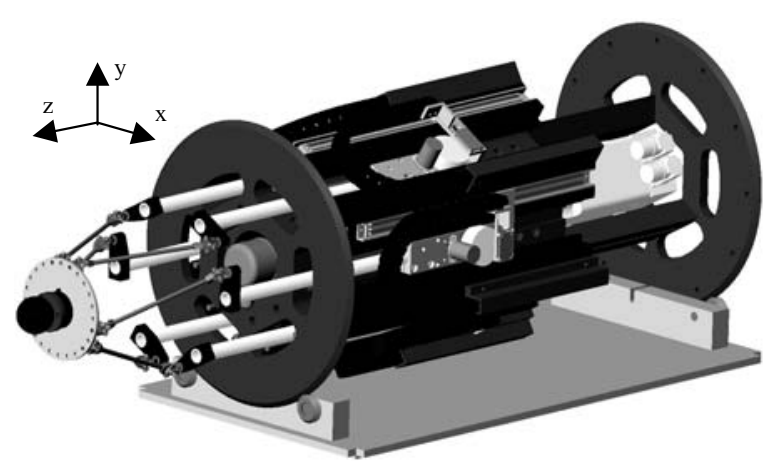

(b) 6 D.O.F. Cobotic Hand Controller.

FIGURE 8. A CAD model of the 6-DOF Cobotic Hand Controller.

Both visual and haptic virtual funnels are tested for aiding telerobotic operation of approaching and aligning the tool to the target position. As shown in Figure 9(A), alignment task was successively performed solely with the aid of visual virtual fixture. However, it was necessary to pay full attention to the visual display during entire operation. In Figure 9(B), a translational trajectory along haptically implemented virtual surface is shown. Once the operator hits

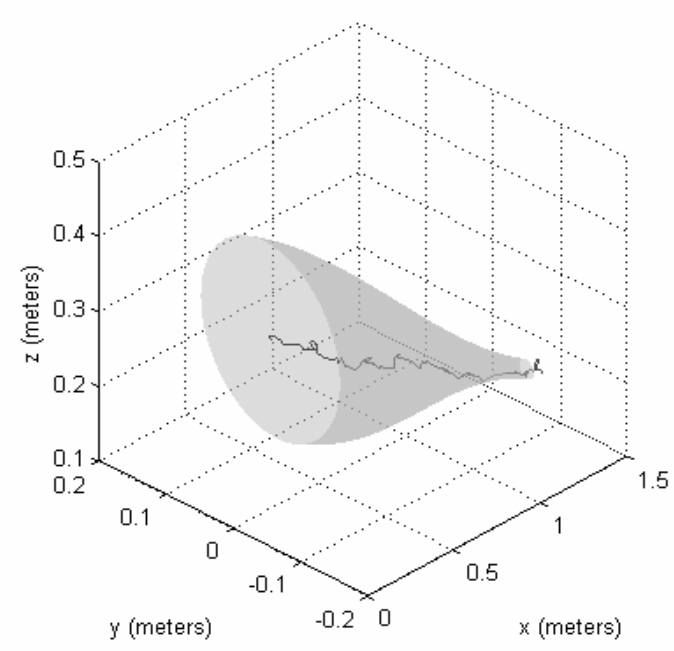

(a) With Visual Virtual Fixture.

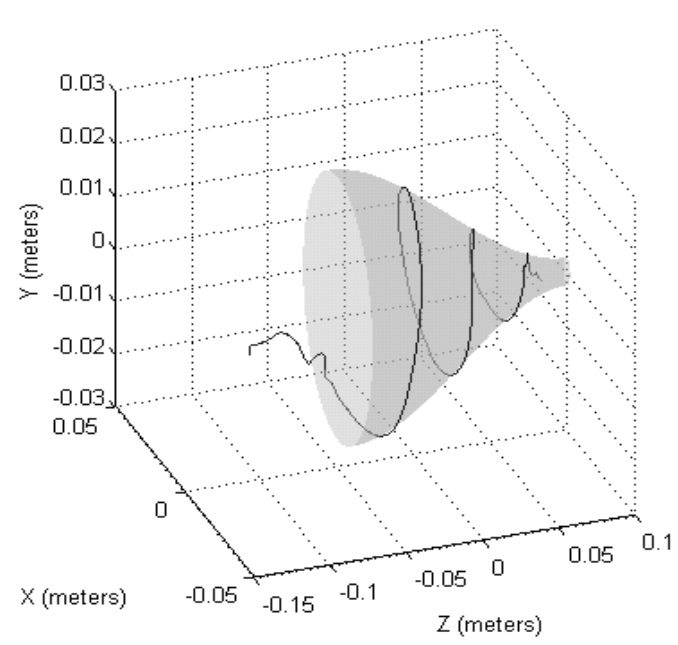

(b) With Haptic Virtual Fixture.

FIGURE 9. Translational Path Taken Along Virtual Funnel. 
the constraint surface, he is able to slide into the target position along the funnel surface. This behavior is contrary to the case of visually augmented teleoperation where the operator tries to keep the current position at the center of funnel. To show the effectiveness of funnel, the operator intended to move around the funnel.

\section{CONCLUSIONS}

Semi-autonomous telerobotic operation technology is introduced consisting of teleautonomy and enhanced operator interface. The presented technology aims at facilitating efficient and precise remote operation of robotic manipulators. Tele-autonomy is accomplished by blending automation and manual operation, in such a way that precise motion is accomplished by the robot while human provides rough motion. By adopting reactive robotic architecture, the system is capable of adapting to unstructured environment. Furthermore, it is possible to build and reconfigure various autonomous behaviors incrementally from motor agents. Development of enhanced operator interface focuses on providing visually and haptically augmented virtual fixture to guides human motion. Building upon Cobot technology, a new hand controller is developed. Experimental operations revealed the effectiveness of each component methods. The proposed technologies originally developed for nuclear facility D\&D may provide effective aid for future space applications where robotic system is expected to be used for handling space structures.

\section{ACKNOWLEDGMENTS}

This research was sponsored by the Environmental Management Science Program of the Office of Environmental Management and Office of Science, U. S. Department of Energy.

\section{REFERENCES}

U.S. Department of Energy, Dual Arm Work Platform Teleoperated Robotics System, Deactivation and Decommissioning Focus Area, Innovative Technology Summary Report:, DOE/EM-0389, 1998.

Noakes, M. W., Love, L. J. and Lloyd, P. D. “Telerobotic planning and control for DOE D\&D operations," in proceedings of IEEE International Conference on Robotics and Automation, 2002.

Park, Y. S., et. al., "Enhanced Teleoperation for D\&D," in proceedings of IEEE International Conference on Robotics and Automation, 2004.

Faulring, E. L., Colgate, J. E., Peshkin, M. A., "A High Performance 6-DOF Haptic Cobot," in proceedings of IEEE International Conference on Robotics and Automation, 2004.

DeJong, B. P., Colgate, J. E., and Peshkin, M. A., "Improving Teleoperation: Reducing Mental Rotations and Translations," in proceedings of IEEE International Conference on Robotics and Automation, 2004.

Arkin, R. C., "Reactive Control as a Substrate for Telerobotic Systems," IEEE Aerospace and Electronics System Magazine, 6, No. 6, pp. 24-31, 1991.

Park, Y. S., "Structured Beam Projection for Semi-automatic Teleoperation," Proc. of SPIE 2000 ISAM Conference on OptoMechatronic Systems, Boston, MA, 2000.

Rosenberg, L. B., "Virtual Fixtures: Perceptual Overlays Enhance Operator Performance in Telepresence Tasks," Ph.D Dissertation, Mechanical Engineering: Stanford University, 1994.

Wannasuphoprasit, W., Akella, P., Peshkin, M., Colgate, J.E., "Cobots: A Novel Material Handling Technology," in proceedings of International Mechanical Engineering Congress and Exposition, Anaheim, ASME 98-WA/MH-2, 1998.

Peshkin, M. A., Colgate, J. E., Wannasuphoprasit, W., Moore, C. A., Gillespie, R. B., and Akella, P., "Cobot Architecture," IEEE Transactions on Robotics and Automation, 17, pp. 377-390, 2001.

Merlet, J. P., "Direct Kinematics and Assembly Modes of Parallel Manipulators.," International Journal of Robotics Research, 11, pp. 150-162, 1992. 